\title{
Fluorescence Polarization Microscopy Enables Spatial Mapping of the 3D Orientation of Piconewton Integrin Traction Forces
}

\author{
Joshua M. Brockman ${ }^{1}$, Aaron T. Blanchard ${ }^{1}$, Khalid Salaita ${ }^{1,2}$, and Alexa L. Mattheyses ${ }^{3 *}$ \\ 1. Wallace H. Coulter Department of Biomedical Engineering, Georgia Institute of Technology and \\ Emory University, Atlanta, Georgia USA \\ 2. Department of Chemistry, Emory University, Atlanta, GA USA \\ 3. Department of Cell, Developmental, and Integrative Biology, University of Alabama at Birmingham, \\ Birmingham, AL USA \\ * Corresponding author: mattheyses@uab.edu
}

Mechanical forces are essential for the survival of many, if not all, living systems. Fundamental biological processes including cell division, migration, and gene expression cannot proceed without molecular level forces $[1,2]$. There are many protein mediated physical and mechanical interactions at the interface between a cell and it's environment, with both other cells and the extracellular milieu. Studying the mechanotransduction, or the interconversion between mechanical forces and chemical signals, of these interactions relies on the development of novel molecular technologies and instrumentation. Developing these tools with high spatial and temporal resolution is paramount to a global understanding of signaling pathways in living systems. Current microscopy-based techniques capable of measuring force direction suffer from $\mu \mathrm{m}$ spatial resolution and $\mathrm{nN}$ force sensitivity, two to three orders of magnitude greater than forces applied by individual receptors [3].

We previously developed molecular tension probes that report the magnitude of individual receptor forces to address these limitations, however in doing so the force orientation was lost [4]. Tension probes are comprised of an extendible "spring-like" element, such as a DNA hairpin, flanked by a fluorophore and quencher. When a receptor transmits $\mathrm{pN}$ forces to the probe, the spring extends, separating the fluorophore from the quencher, and generating up to a 100-fold increase in fluorescence. While molecular tension probes improve spatial resolution and force sensitivity, they provide no information about force orientation. No current technique is able to measure both the magnitude and direction of molecular forces at the piconewton scale.

To fill the gap, we integrated fluorescence polarization microscopy and molecular tension probes to measure the magnitude and 3D orientation of $\mathrm{pN}$ cellular forces. Because fluorophores absorb and emit polarized light in an orientation-dependent manner, fluorescence polarization microscopy can be used to calculate orientations from measurable quantities such as fluorescence intensity [5]. Here we demonstrate, that cyanine dye tagged DNA hairpin based molecular tension probes can be used in combination with fluorescence polarization - what we named molecular force microscopy (MFM) - to determine the orientation of cellular forces (Figure 1) [6].

We will describe the experimental system, utlizing excitation resolved fluorescence polarization microscopy to determine the orientation of cellular traction forces reported by molecular tension probes. There are several key features that will be described theoretically and experimentally. First, it is critical that the dye stack rigidly with the DNA of the tension probe, thereby ensuring the fluorophore reports the probe orientation. Second, the mechanical force required to open the tension probe will also orient it, 
allowing the probe to report the force direction. Third, only open probes, or those experiencing a mechanical force, contribute substantially to the fluorescence signal. Therefore the signal is dominiated by probes oriented by a force with minimal background - what we term mechanoselection. The sensitivity, accuracy, and limitations of MFM will be explored with computational modeling.

We will next present the experimental results from two model systems using MFM. First we determined the orientation of traction forces generated by integrin $\alpha_{\mathrm{IIb}} \beta_{3}$ in human platelets, chosen because their activation and clotting functions are mediated by mechanical forces. Multi-cell analysis revealed two populations of platelets with differentially organized forces. The diffraction limited spatial resolution of MFM was critical in discerning these differences in spatial orientation. Next we mapped integrinmediated forces in NIH-3T3 fibroblasts. We were able to determine the 3D organization of integrin forces were relatively well aligned relative to the cell and to individual focal adhesions.

Finally, we will discuss technical advances, beyond linear dichroism, to our polarization microscopy methods to greatly improve time and orientational resolution. Due to its simplicity and compatibility with fluorescence microscopy, we anticipate MFM will provide a powerful link between structural biology and mechanobiology [7].

\section{References:}

[1] V Vogel and M Sheetz, Nat. Rev. Mol. Cell Biol. 7, (2006) 265.

[2] C DuFort, M Paszek, and V Weaver, Nat Rev Mol Cell Biol 12, (2011) 308.

[3] W Polacheck and C Chen, Nat Methods 13, (2016) 415.

[4] D Stabley et al., Nat Methods 9, (2012) 64.

[5] S Bradley et al., Biophys J 101, (2011) 985.

[6] J Brockman et al., Nat Methods 15 (218) 115.

[7] The authors acknowledge funding from NIGMS R01 GM124472 (K.S.), NSF 1350829 (K.S.), NSF

CAREER 1553344 (A.L.M.) and NSF IDBR 1353939 (K.S. and A.L.M.).
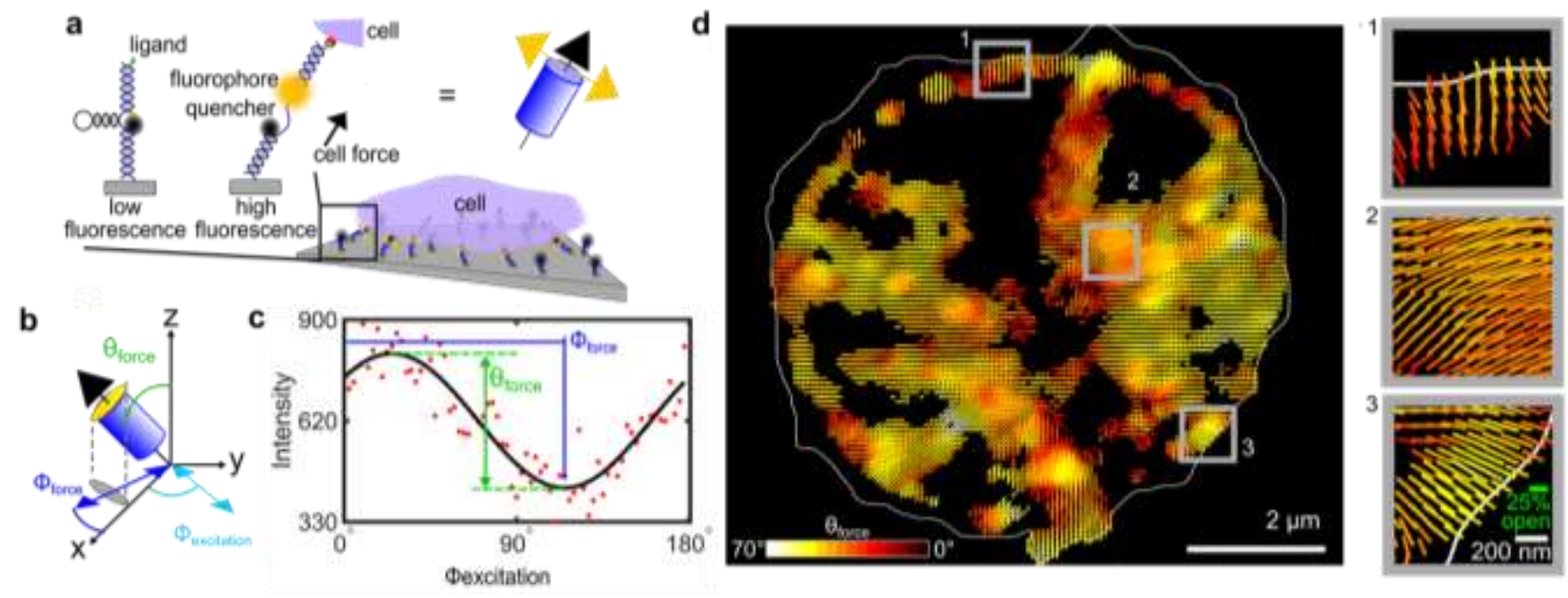

Figure 1: a) Molecular tension probes are surface-anchored DNA hairpins flanked by a fluorophorequencher pair that unfold and dequench when pulled on with force exceeding a sequence-specific threshold. b) The de-quenched fluorophore rigidly stacks perpendicular to the opened tension probe. It's polar $\left(\theta_{\text {force }}\right)$ and azimuthal ( $\left.\varphi_{\text {force }}\right)$ angles can be inferred by imaging with polarized excitation light, rotating the polarization angle, and c) fitting the intensity to a $\cos ^{2}$ function. d) Applying this treatment to each pixel in a widefield image Allows diffraction-limited force orientation mapping. 